\title{
Research Paper \\ Effects of an 8-Week Aerobic Exercise Program on Some Indicators of Oxidative Stress in Elderly Women
}

\author{
*Keyvan Hejazi' 9 , Mahdi Ghahremani Moghaddam² $\odot$, Teimour Darzabi
}

1. Department of Physical Education, Toos Institute of Higher Education, Mashhad, Iran.

2. Department of Exercise Physiology, Faculty of Sport Sciences, Ferdowsi University of Mashhad, Mashhad, Iran.

3. Department of Vocational Science, Faculty Technical of Shahid Montazeri, Technical and Vocational University, Mashhad, Iran.

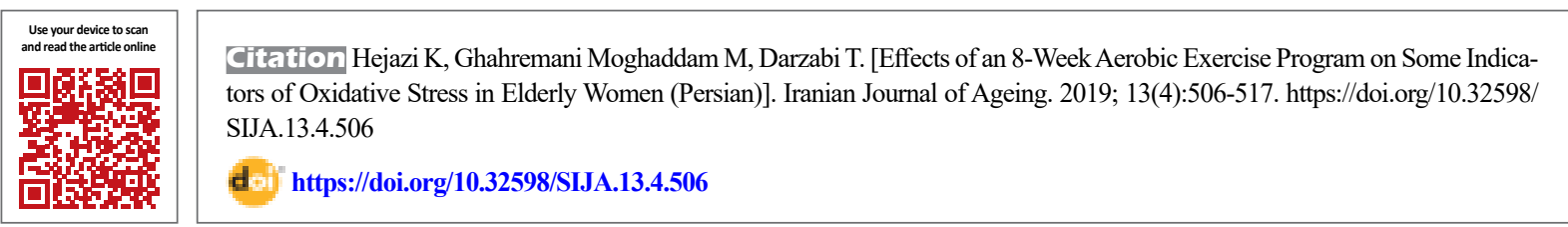

(c) $0(3)$

Received: 01 Aug 2018 Accepted: 17 Oct 2018 Available Online: 01 Jan 2019

Keywords: Aerobic exercise, 8-Hydroxy-2'deoxyguanosine, Elderly

\section{A B STRACT}

Objectives Oxidative stress has an important role in the pathogenesis of diseases such as cardiovascular disease, cancer, diabetes and aging. The present study investigated the effects of 8 weeks of aerobic training on 8-hydroxy-2'-deoxyguanosine and body composition in elderly women.

Methods \& Materials This was a quasi-experimental study. The study participants were selected by convenience sampling method. In total, 21 subjects with the age range of $60-70$ years were assigned into 2 groups (experimental $[n=11]$ and control $[n=10]$ ). The 8-week aerobic training program were demonstrated 3 sessions a week, for $45-60$ minutes per session, with the intensity of $50 \%$ to $70 \%$ of maximum heart rate. The blood samples were obtained 24 hours after the intervention to measure the serum levels of $8-0 H d G$. For comparison of within and between group mean scores, Paired t test and Independent samples t test were used, respectively.

Results Eight weeks aerobic training significantly reduced the weight, BMI and body fat percentage in elderly women. Moreover, the levels of serum 8-OHdG after an 8 weeks aerobic training significantly reduced. However, the levels of 8-OHdG urine reduced at the end of the training, but it was non-significant. There were significant differences between active and inactive elderly woman in terms of weight and serum 8-OHdG variables.

Conclusion This study suggests that aerobic training decreases serum 8-OHdG. Regular aerobic physical activity with moderate intensity improves the body anti-oxidative capacity and can prevent the incidence of atherosclerosis disease.

\section{Extended Abstract}

\section{Objectives}

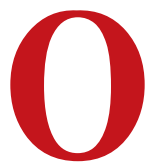

xidative stress plays an important role in the pathogenesis of aging and some diseases such as cardiovascular disease, cancer, and diabetes. Production of various types of oxygen causes biological damage, and potentially exacerbates these complications [1-2] There is a close relationship between the production of Reactive Oxygen Species (ROS) and the analysis of antioxidant systems [1]. Structural changes in DNA as a result of exposure to ROS, can accelerate aging, and cause atherosclerosis and diabetes [3]. One of the factors that has been studied in recent years is 8-hy-

\section{* Corresponding Author:}

Keyvan Hejazi, PhD.

Address: Department of Physical Education, Toos Institute of Higher Education, Mashhad, Iran.

Tel: +98 (915) 1253513

E-mail: keyvanhejazi@gmail.com 
droxy-2'-deoxyguanosine (8-OHdG), which is an index of oxidative DNA damage [4]. The positive role of exercise and physical activity in the prevention of cardiovascular diseases have been already proven [5]. The current study examined the effect of an 8-week aerobic exercise program on $8-\mathrm{OHdG}$ level and body composition of inactive elderly women.

\section{Methods and Materials}

In this quasi-experimental study, 21 elderly women with the mean age of 60-70 years, and Body Mass Index (BMI) of $29-30 \mathrm{~kg} / \mathrm{m}^{2}$ participated. The samples were selected using convenience and purposeful sampling methods. The subjects were then randomly divided into test $(n=11)$ and control $(n=10)$ groups. Aerobic training were held for 8 weeks; 4 sessions per week. The duration of each session was 45-60 minutes. The intervention program for those in test group included: 1. A 10-minute warm-up; 2. Aerobic training for $45-60$ minutes with an intensity of $50-70 \%$ of the maximum heart rate. The training time gradually increased from 30 minutes at the beginning to 45 minutes at the end; and 3. A 10-minute cool-down. The intensity of training was measured by a heart rate sensor (Polar, Finland).
The blood samples were collected 24 hours before initiating the training and 24 hours after the last training session to measure the serum and urine levels of 8-OHdG. To determine the 8-OHdG level, ELISA method was employed using related assay kit (Cusabio, Japan). Bioelectrical impedance analysis was used for estimating the body composition of the samples. SPSS was used for data analysis. In addition, within group and between group comparisons were performed using Paired t test and Independent $t$ test, respectively. The significance level was set at $\mathrm{P}>0.05$. This study was a registered project (code: 2.32123 ), which was conducted with the financial support of the Vice Chancellor of Research and Technology Department of Ferdowsi University of Mashhad.

\section{Results}

According to the obtained results, there were no significant differences between the test and control groups in terms of height, weight, BMI, body fat percentage, and the serum and urine levels of 8-OHdG. The obtained results revealed that an 8 -week aerobic training program significantly reduced Mean \pm SD scores of body weight from $68.68 \pm 10.44$ to $67.48 \pm 10.23 \mathrm{~kg}(\mathrm{P}=0.001)$, BMI Mean \pm SD scores from $29.52 \pm 3.37$ to $28.83 \pm 3.23 \mathrm{~kg} /$ $\mathrm{m}^{2}(\mathrm{P}=0.01)$, and body fat percentage Mean \pm SD scores

Table 1. Between-group and within-group comparisons of changes in body composition and the serum and urine levels of $8-\mathrm{OHdG}$

\begin{tabular}{|c|c|c|c|c|c|}
\hline \multirow{3}{*}{ Variables } & \multirow{3}{*}{ Groups } & \multicolumn{2}{|c|}{ Mean $\pm S D$} & \multicolumn{2}{|c|}{ Changes } \\
\hline & & \multirow{2}{*}{ Pre-Test } & \multirow{2}{*}{ Post-Test } & Within Group* & Between Group** \\
\hline & & & & $\mathbf{P}$ & $\mathbf{P}$ \\
\hline \multirow{3}{*}{ Weight, kg } & Test & $68.68 \pm 10.44$ & $67.48 \pm 10.23$ & $0.00^{* * *}$ & \multirow{3}{*}{$0.00^{*}$} \\
\hline & & & & & \\
\hline & Control & $71.79 \pm 10.40$ & $72.13 \pm 9.86$ & 0.27 & \\
\hline \multirow[b]{2}{*}{$\mathrm{BMI}, \mathrm{kg} / \mathrm{m}^{2}$} & Test & $29.52 \pm 3.37$ & $28.83 \pm 3.23$ & $0.01^{*}$ & \multirow[b]{2}{*}{0.06} \\
\hline & Control & $30.18 \pm 4.02$ & $30.13 \pm 3.93$ & 0.84 & \\
\hline \multirow{3}{*}{ Body fat, $\%$} & Test & $43.20 \pm 6.98$ & $41.36 \pm 7.24$ & $0.02^{*}$ & \multirow{3}{*}{0.06} \\
\hline & & & & & \\
\hline & Control & $41.51 \pm 6.35$ & $41.38 \pm 6.73$ & 0.80 & \\
\hline \multirow{2}{*}{$\begin{array}{l}\text { 8-OHdG serum level,ng/ } \\
\mathrm{mL}\end{array}$} & Test & $329.00 \pm 191.81$ & $271.00 \pm 143.34$ & $0.012^{*}$ & \multirow{2}{*}{$0.048^{*}$} \\
\hline & Control & $366.20 \pm 146.92$ & $422.50 \pm 97.76$ & 0.273 & \\
\hline \multirow{2}{*}{$\begin{array}{l}\text { 8-OHdG urine level, } \mathrm{ng} / \\
\mathrm{mL}\end{array}$} & Test & $8.14 \pm 4.17$ & $6.01 \pm 1.08$ & 0.118 & \multirow{2}{*}{0.305} \\
\hline & Control & $14.61 \pm 11.40$ & $13.16 \pm 9.23$ & 0.591 & \\
\hline
\end{tabular}

** Paired t-test; *** Independent $\mathrm{t}$ test, * Significant at $\mathrm{P}<0.05$ 
from $43.20 \pm 6.98$ to $41.36 \pm 7.24(\mathrm{P}=0.02)$ in the samples. Reduction in the Mean \pm SD scores of serum levels of 8 -OHdG from $329 \pm 191.81$ to $271 \pm 143.34 \mathrm{ng} / \mathrm{mL}$ was significant at the end of intervention $(\mathrm{P}=0.012)$. However, reduction in the Mean \pm SD scores of urine levels of 8-OHdG from $8.14 \pm 4.17$ to $6.01 \pm 1.08 \mathrm{ng} / \mathrm{mL}$ was not significant $(\mathrm{P}>0.05)$.

The Independent $t$ test results regarding betweengroup comparison of post-test mean scores revealed significant differences between the 2 groups in terms of body weight $(\mathrm{P}=0.01)$ and the serum levels of $8-\mathrm{OHdG}$ $(\mathrm{P}=0.04)$. However, in terms of $\mathrm{BMI}(\mathrm{P}=0.06)$, body fat percentage $(\mathrm{P}=0.06)$, and the urine levels of 8-OHdG ( $\mathrm{P}=0.305)$, no significant differences were observed between them (Table 1). In other words, the aerobic exercise significantly reduced the serum levels of 8-OHdG in the test group. Therefore, an 8-week aerobic training program had a significant effect on the serum level of 8-OHdG.

\section{Conclusion}

Given the effect of 8 weeks of aerobic training on reducing weight, BMI, and the serum level of 8-OHdG in elderly women, It can be concluded that aerobic exercise is an appropriate treatment for the elderly women. In addition, due to the significant reduction in the serum levels of 8-OHdG, regular aerobic exercises with moderate intensity improved antioxidant function. However, considering the important role of physical activity in the prevention and treatment of many diseases, and increasing the sense of satisfaction in the elderly, specialists should suggest a combination of pharmacotherapy, training and nutritional counseling for the treatment of cardiovascular diseases. Thus, we recommend aerobic exercises to prevent the adverse effects of increased atherosclerosis, and it can be considered as a vital part in the lifestyle of inactive elderly women.

\section{Ethical Considerations}

\section{Compliance with ethical guidelines}

This study is a research project registered at Ferdowsi University of Mashhad (code: 2.32123).

\section{Funding}

This study has received financial support from Ferdowsi University of Mashhad.

\section{Authors contributions}

Conceptualization and investigation: Keyvan Hejazi, Mahdi Ghahremani Moghaddam, and Teimour Darzabi; Editing: Keyvan Hejazi; Project administration: Keyvan Hejazi and Mahdi Ghahremani Moghaddam; Funding acquisition: Mahdi Ghahremani Moghaddam.

\section{Conflict of interest}

The authors declared no conflict of interest. 


\title{
اثر هشت هفته تمرينات هوازى بر برخى شاخصهاى استرس اكسيداتيو زنان سالمند
}

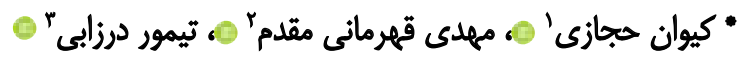 \\ ا - كروه تربيت بدنى، مؤسسه آموزش عالى توس، مشهيد، ايران.

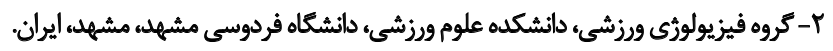

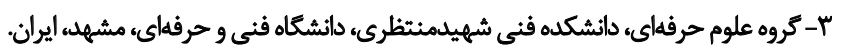

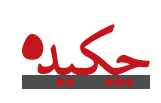

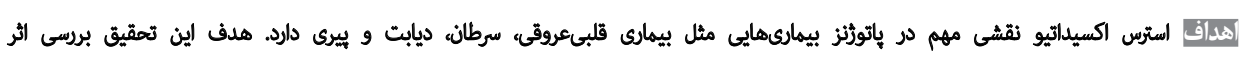

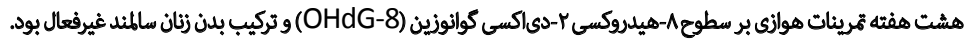

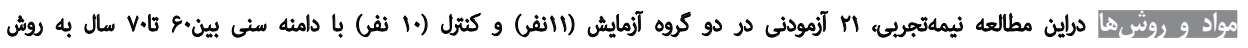

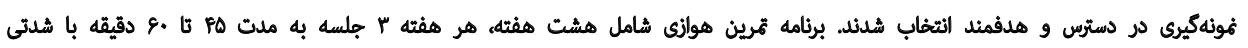

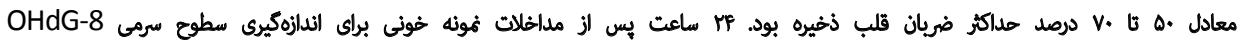

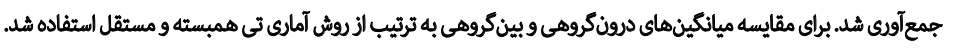

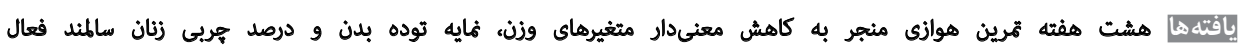

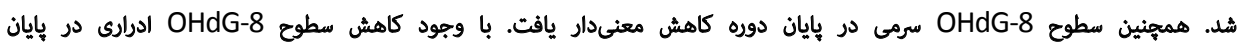

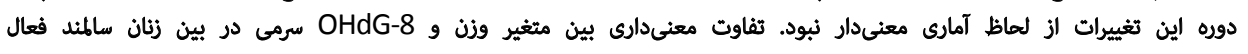
و غيرقعال مشاهده شد.

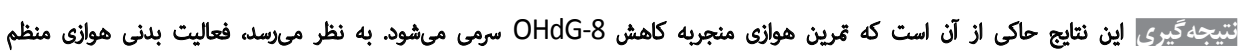

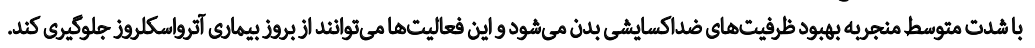

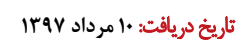

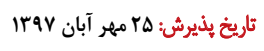
تاريخ انتشار: الدى

كليدوازهها:

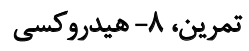
ب- دى اكسى كوائوزين، سالمند

تحميل مىكند [ه]]. از لحاظ اقتصادى هزينههاى مرتبط با باين

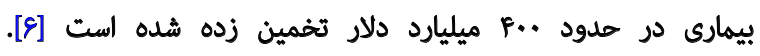

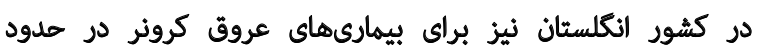

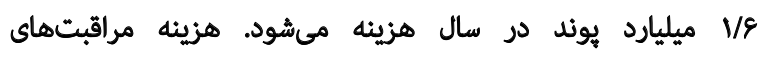

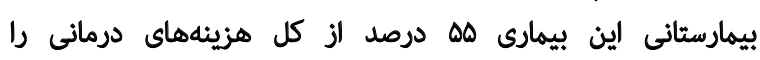
تشكيل مىدهند [V]

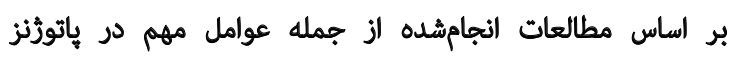

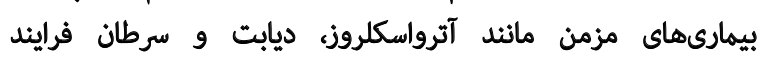

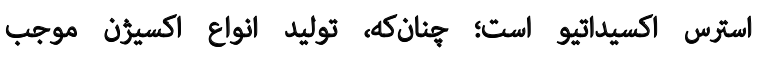

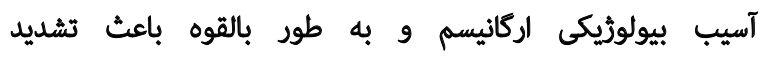

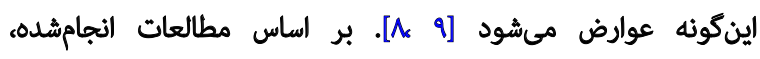

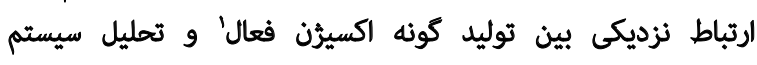

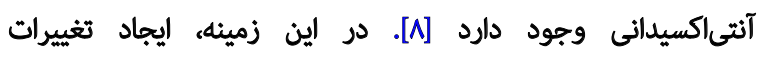

1. Reactive Oxygen Species (ROS)

doves

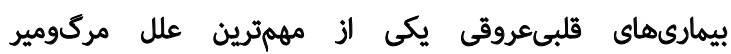

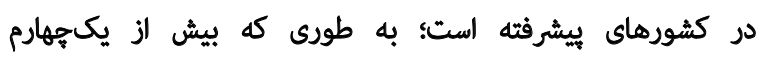

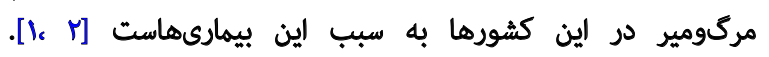

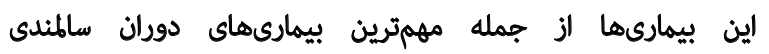

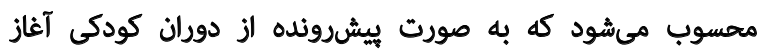

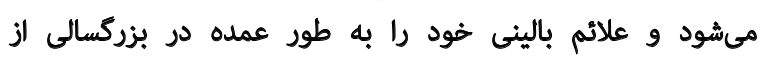

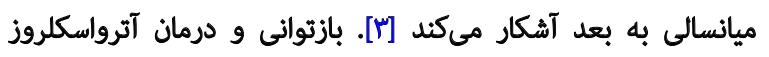

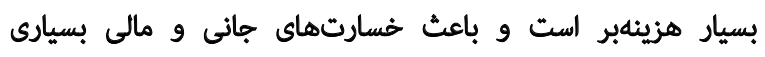

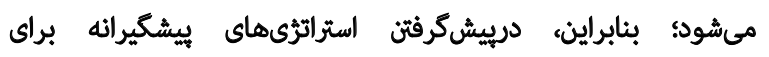
اين بيمارى ضرورى است [F]

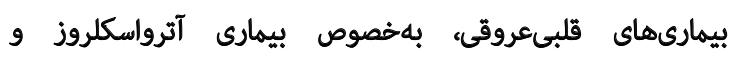

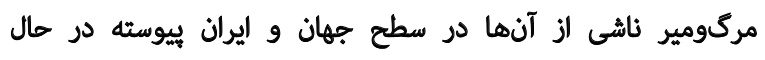
افزايش است و اين بيمارى هزينههاى درمانى سنكينى بر كشورها

$$
\begin{aligned}
& \text { : نويسينده مسينول: } \\
& \text { دكتر كيوان حجازى مئول }
\end{aligned}
$$

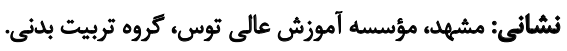

$$
\begin{aligned}
& \text { تلفن: } \\
& \text { يست الكترونيكى: keyvanhejazi@gmail.com }
\end{aligned}
$$




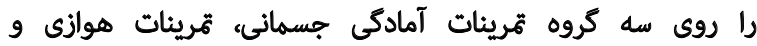

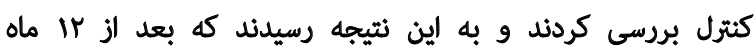

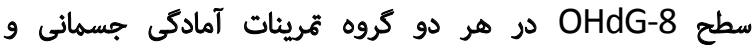

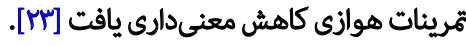

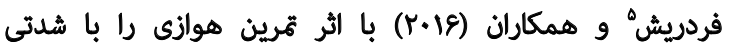

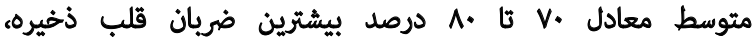

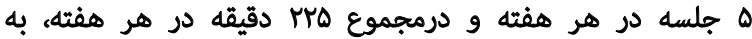

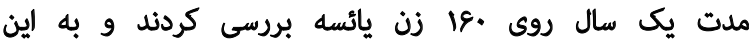

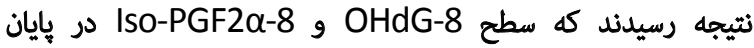

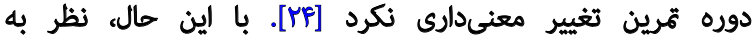

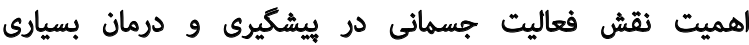

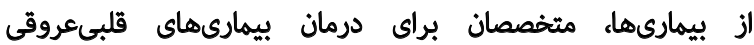

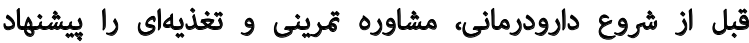

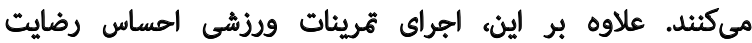

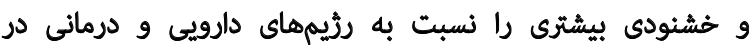

افراد ايجاد مىكند.

در اين زمينه، با توجه به تحقيقات محدود در رابطه با تأثير فعاليت

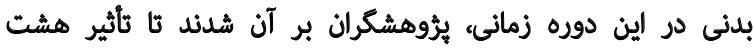

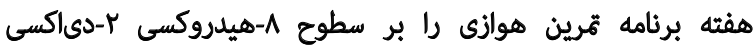
كوانوزين و تركيب بدن زنان سالمند غيرفعال بررسى كنثل.

$$
\text { dollbo }
$$

اين تصقيق الز نوع نيمهتجربى است كه دو كروه آزمايش با

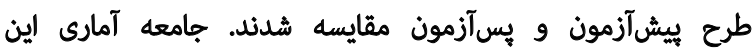

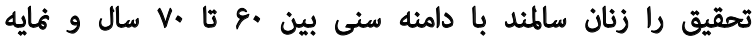

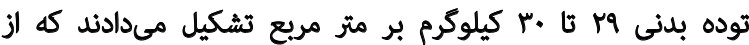

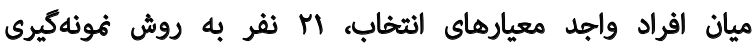
دردسترس و هدفدار كزينش و به دو كروه آزمايش (11 نفر) و و كنترل (· انفر) تقسيم شدند.

در مرحله ذضِّت افراد با ماهيت و نحوه همكارى با اجراى

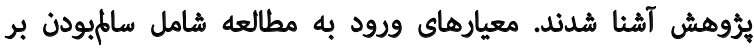

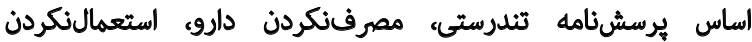

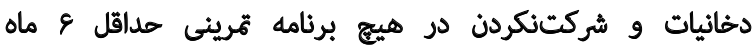

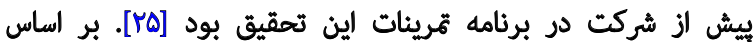

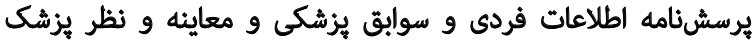

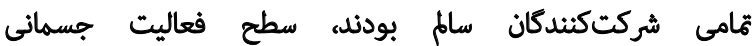

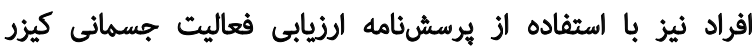

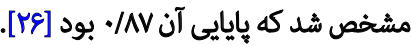
آزمودنىها بر اسياس شرايط تحقيق به صورت داوطلبانه در

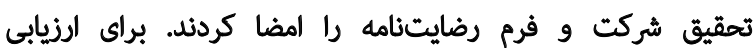

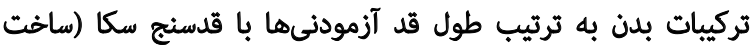

5. Friedenreich
ساختارى دNA در نتيجه قراركرفتن در معرض كونه اكسيرن

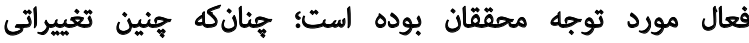
نقش مهمى در بروز بيرى و بيمارىهاى آترواسكلروز و ديابت هودهان

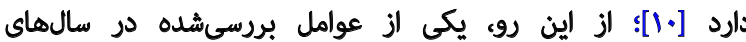

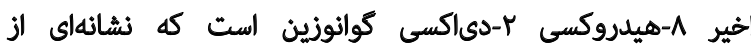

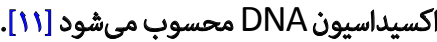

در اثر فشار اكسايشى، بسيارى از ماكرومولكولها آسيب

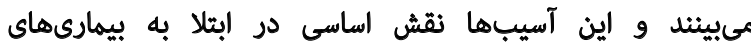

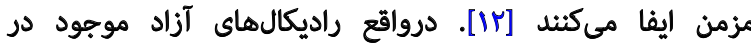
يلاسها به جربىهاى خون همله همله كرده و موجب الكسيداسيون

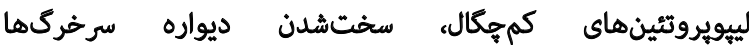
و درنهايت، بروز بيمارىهاى قلبىعروقى مانئد آترواسكلروز

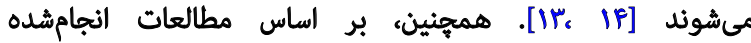

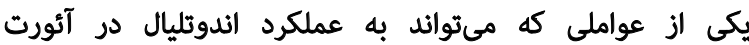

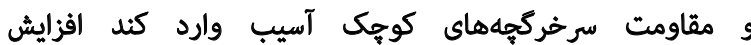

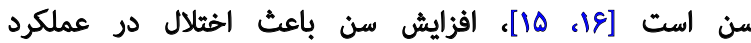

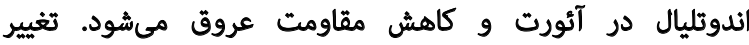

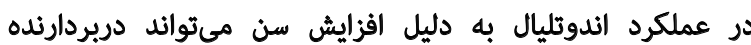
علائم بيمارىهاى قلبىعروقى باشد [IV]

امروزه آثار مثبت تمرين و فعاليت بدنى براي ييشكيرى الز

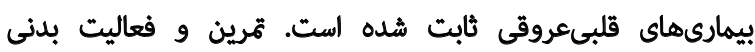

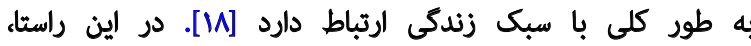

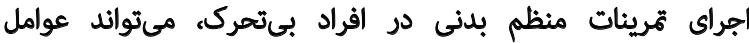

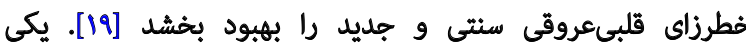

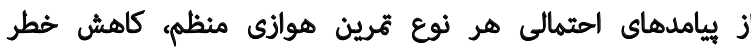

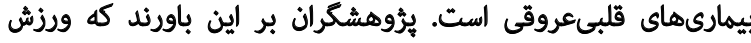

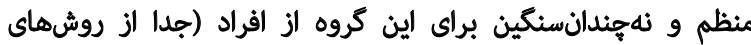

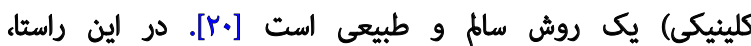

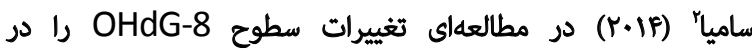

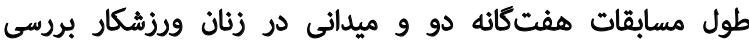
كرد و به اين نتيجه رسيد كه سطوح مالون دي آلدوئيد و -OHdG

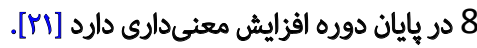

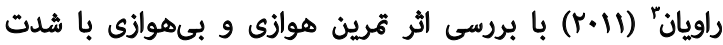

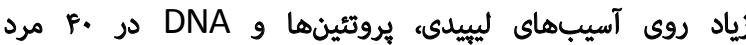

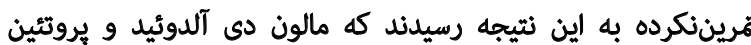

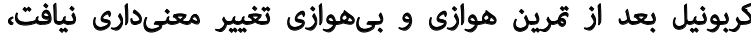

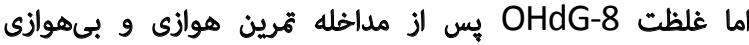

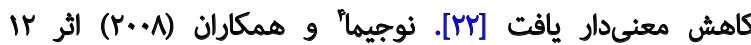

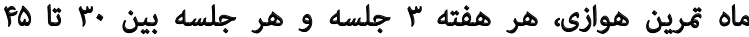

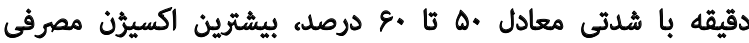


انجام و دادها جمعآورى شد. همجنين، شدث ثمرين علاوه

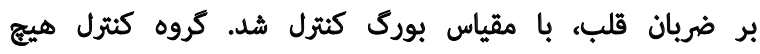

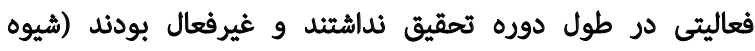

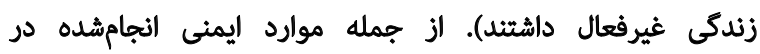

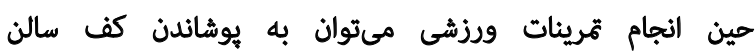

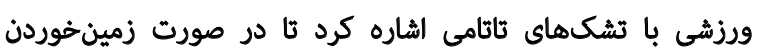

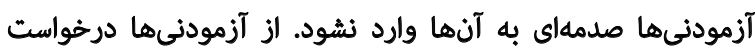

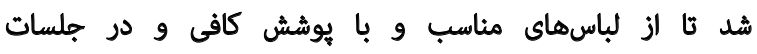
تمرينى از كفش مناسب ورزشى استفاده كنثد.

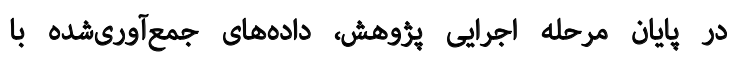

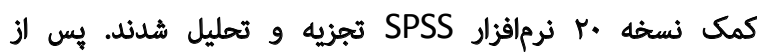

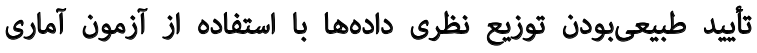

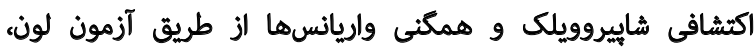

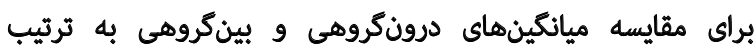

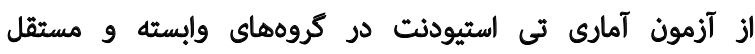

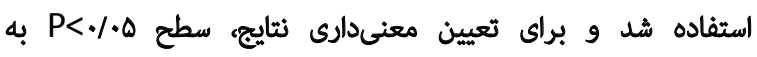
عنوان ضابطه تصميم يرى در نظر كرفته شد.

Lavill

مشخصات آزمودنىهاي كروه آزمايش و كنتل در هدول هدول

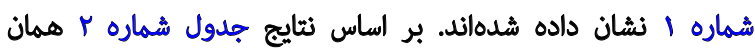

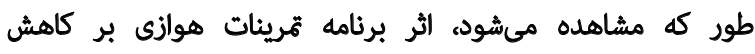

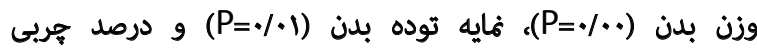
بدئى (Y+/

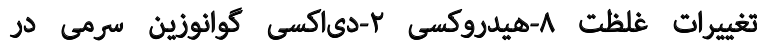

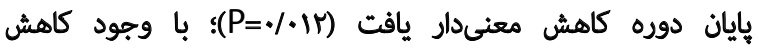

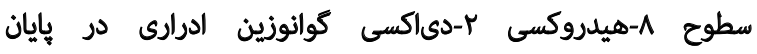

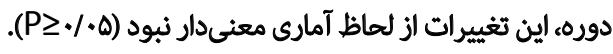
بر اساس يافتههاى جدول شماره r ب، تغييرات ميانكينهاى

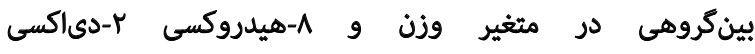
كوانوزين سرمى در بين زينان ساطلند فعال و غير غيرفعال مشاهده

شد (P) (P) (
كشور آلان) با حساسيت ه ميليمتر، محيط باسن و كمر با متر

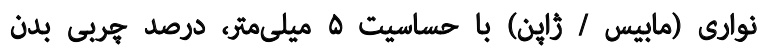

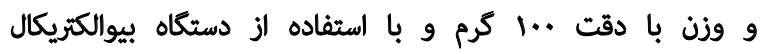

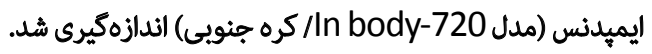
الز تقسيم محيط كمر به محيط باسن، نسبت دور كمر به باسن

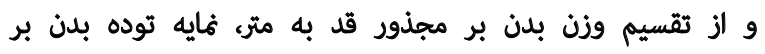

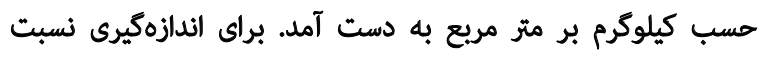

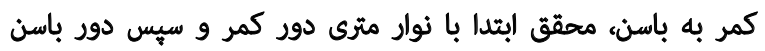

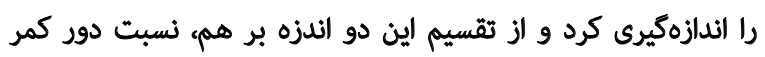

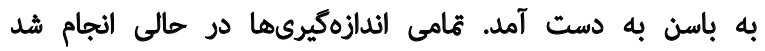

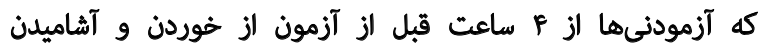

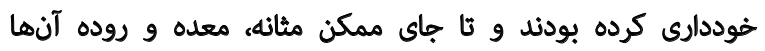

تخليه شده بود.

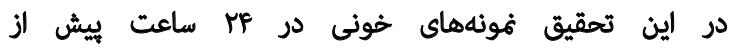

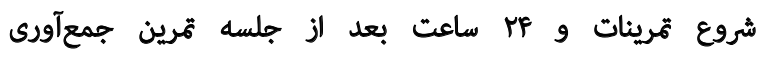

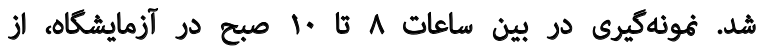
سياهرى دست هي هر آزمودنى، در وضعيت نشسته و در در حالت

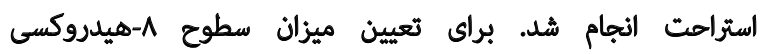

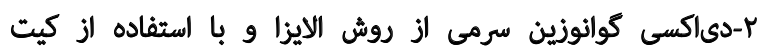
كازابايو، ساخت ثراين انجام شداند.

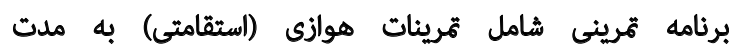

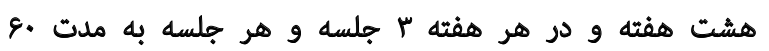

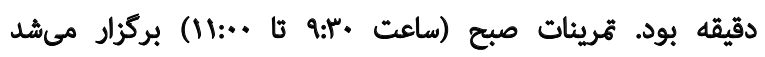

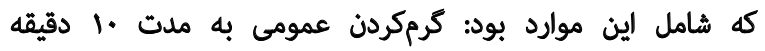

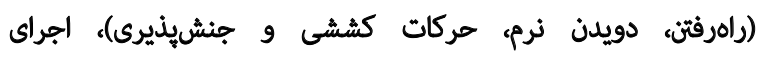

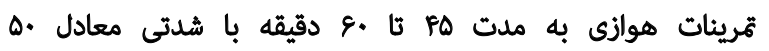

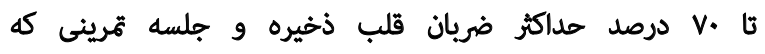

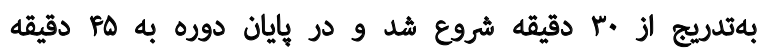

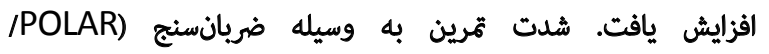
فنلاند) كنترل شـد.

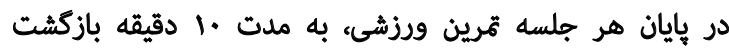

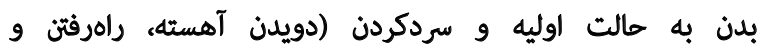

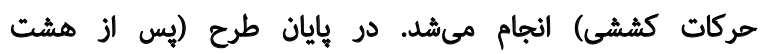

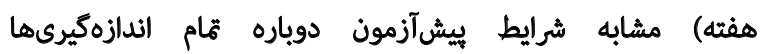

جدول ا. ويرُكى هاى آزمودنى هاى شركت كثنده در مطالعه

\begin{tabular}{|c|c|c|c|c|}
\hline \multicolumn{4}{|c|}{ مياتكين لانحراف معيار } & \multirow{2}{*}{ 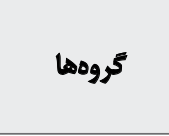 } \\
\hline نمايه توده بدن (كيلوكرم/متر مريع) & وزن (كيلوكرم) & قد (سانتىمتر) & سن (سال) & \\
\hline$r q / \Delta T \pm T / T r$ & 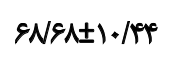 & $\mid \Delta r / q r \pm \Delta / r$. & $g F / r q \pm F / 1 r$ & أزمايش (Iانفر) \\
\hline$r \cdot / M \pm F / . r$ & $V \cdot / v 9 \pm 1 \cdot / r$ & $\mid \Delta f / \Lambda \cdot \pm V / V^{e}$ & $g \& / V \cdot \pm r / W$ & كتثرل (·. نفر) \\
\hline
\end{tabular}


جدول r. مقايسه تغييرات واريانس درون كروهى و بين كروهى در سطوح ^-هيدروكسى Y-دىاكسى گوانوزين سرمى زئن سالمند غيرفعال

\begin{tabular}{|c|c|c|c|c|c|}
\hline \multicolumn{2}{|c|}{$\mathbf{P}$} & \multicolumn{2}{|c|}{ ميانكين } & \multirow{2}{*}{ 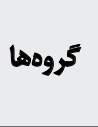 } & \multirow{2}{*}{ متغيرها } \\
\hline بين كروه & درون كروه & يسأزهون & بيشيآزمون & & \\
\hline \multirow{2}{*}{$\% *^{*}$} & $1+*^{*}$ & SV/ศAさ1./Mr & SNGN士1+/RF & آزمايش & \multirow{2}{*}{ (كيلوكرم) } \\
\hline &.$/ \mathrm{rV}$ & VT/KIV/N & $r / v a \pm 1 . / \varphi$. & كنترل & \\
\hline \multirow{2}{*}{.1 .9} & & & & آزمايش & \multirow{2}{*}{ (كيلو كرم برمتر مربح) } \\
\hline & - /AF & $r+/ r \pm \Psi / q \mu$ & $r \cdot / N \pm+/ \cdot r$ & كنترل & \\
\hline \multirow{2}{*}{+1.9} & $H \cdot r^{*}$ & $F \mid / F E \pm V / T \varphi$ & $r w / r+ \pm s / q u$ & آزمايش & \multirow{2}{*}{ 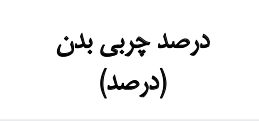 } \\
\hline & $+/ \Lambda$ & $\varphi \backslash / \mu \Delta \pm / n^{\prime}$ & $f \mid / \Delta I \pm \varepsilon / N \Delta$ & كنترل & \\
\hline \multirow{2}{*}{$\cdot 1 \cdot 4 \lambda^{*}$} & $\cdot / \cdot 1 r^{*}$ & $r Y I / * \pm \pm I r T / M f$ & $M T q / \cdot \pm \pm \mid q 1 / 11$ & آزمايش & \multirow{2}{*}{ 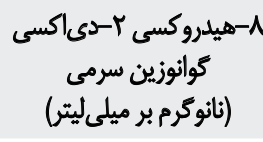 } \\
\hline &.$/ r n$ & 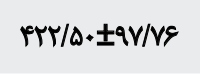 & reg/r.土lfeg/ar & كنترل & \\
\hline \multirow{2}{*}{$\cdot / \mu \cdot \Delta$} &.$/ M$ & $8 / \cdot 1 \pm 1 / * 1$ & $N / F \pm F / I V$ & آزمايش & \multirow{2}{*}{ 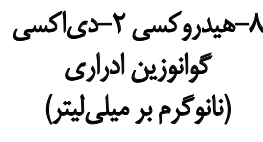 } \\
\hline & $\cdot / \Delta 91$ & $|r /| q \pm q / T$ & $|f / 8| \pm \mid 1 / 4$ & كنترل & \\
\hline
\end{tabular}

$P<\cdot 1+\Delta$ ه * معنى دارى در سطح *

مىتواند نوع فعاليث باشدء زيرا دليل فيزيولوزي هنين

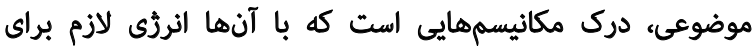

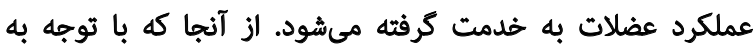

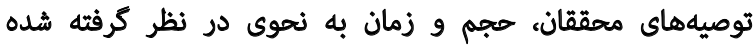

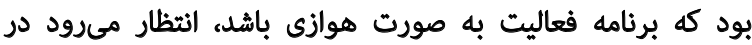

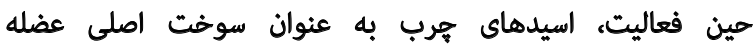

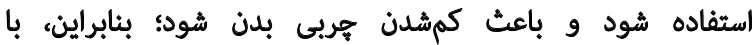
توجه به ماهيت تحقيق كه هوازى است، ميتوان كفت كهن كهان

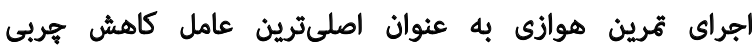

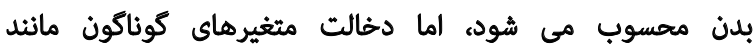

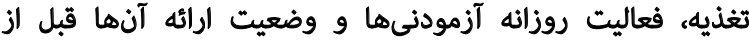

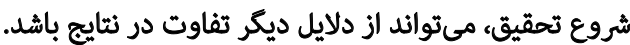
ثأثير تمرينات ورزشى هوازى بر تركيب بدن، الفزايش قابليت

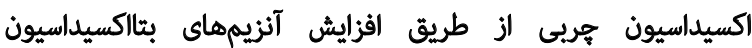

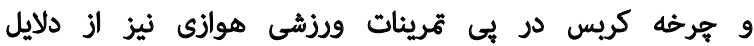

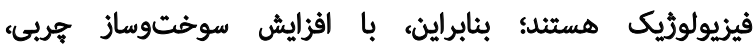

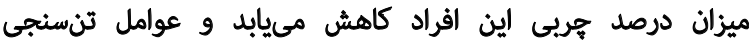

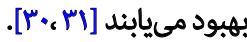

يافتهاى تمقيق نشان داد 1 هفته تمرينات هوازى منجر به كاهش معنى

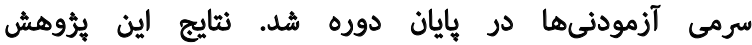

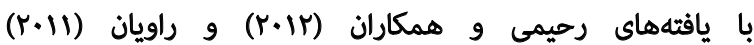

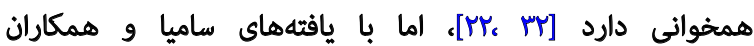

ث

هدف از اين مطالعه بررسى تأثير 1 هفته ثمرين هوازى بر

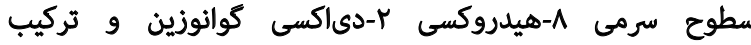
بدن زنان سالمثن غيرفعال بود. بر اساس نتايج بهدستآمده ازئرئ

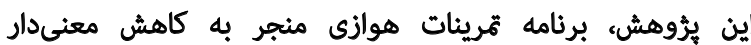

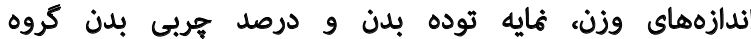

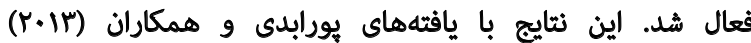

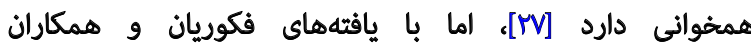

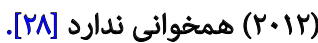

يورابدى و همكاران (r+r) با بررسى \& هفته تمرينات اينتزوال

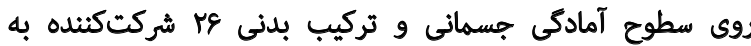

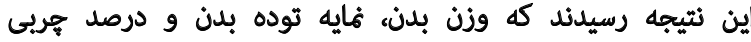

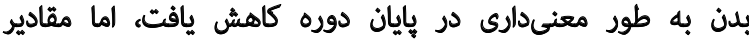

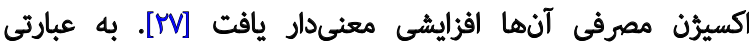

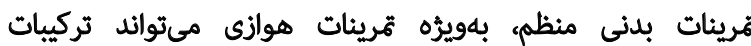

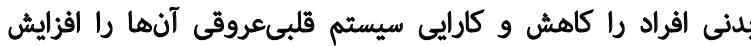

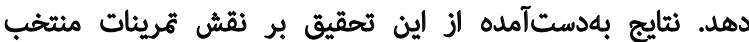

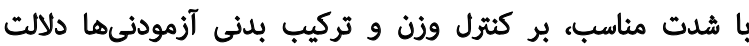

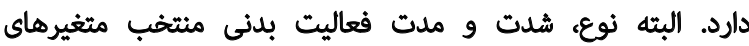

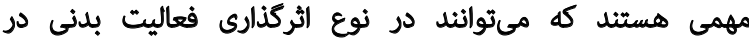

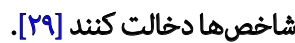

در اين خصوص، دلايل مختلفى براي الختلاف بين نثايج

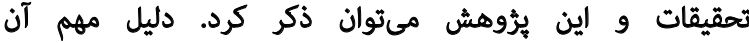


عمل كنند؛ مكانيسمهايى همانثد برداشت اكسيرن يا كاهش يوائ

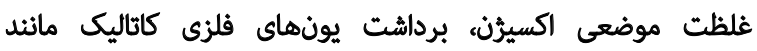

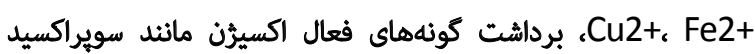

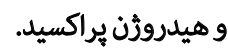

قطعكردن واكنشهاى زنجيرهاى و و به طور كلى قابليت

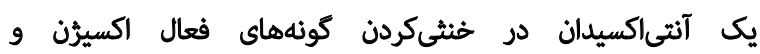

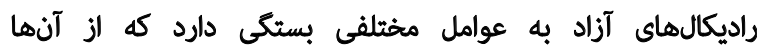

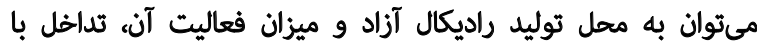

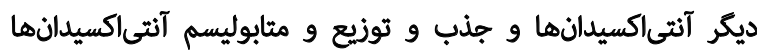

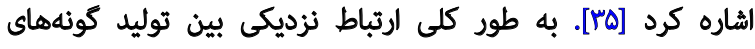
اكسيرن واكنشيذير و شدت ورزش وجود دارد [عزب].

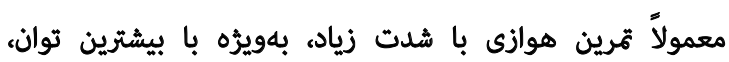

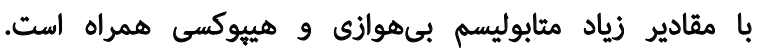

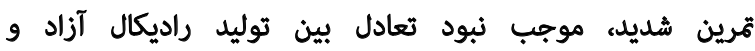

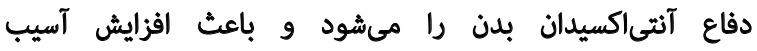

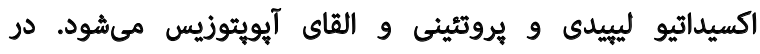

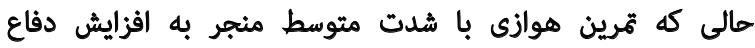

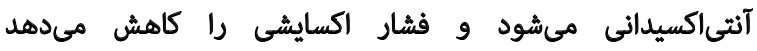

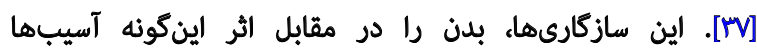

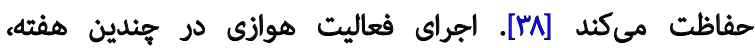

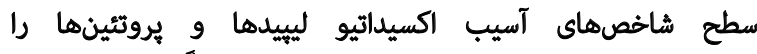

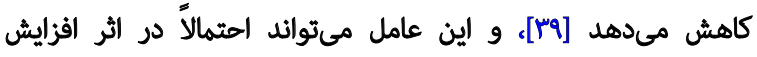

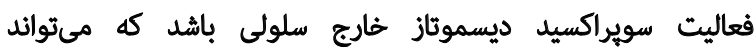

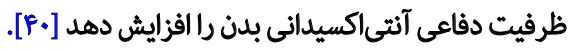

\section{نتيجلَيرى نمهايى}

به طور كلى با توجه به كاهش وزنه نمايه توده بدن، درصد

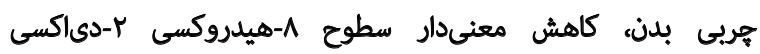

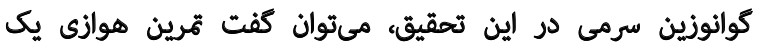

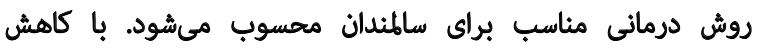

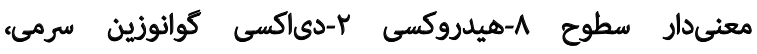

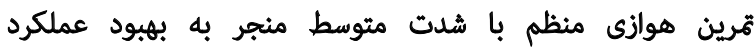

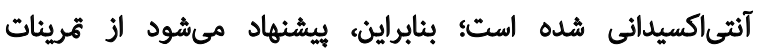

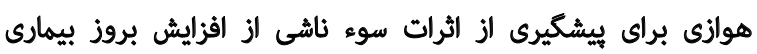

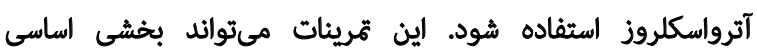
در سبك زندكى افراد سالمئد غيرفعال باشد.

با توجه به اينكه اين مطالعه با محدوديتهايى همجيون رزيم

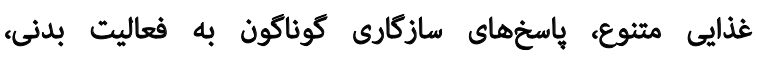

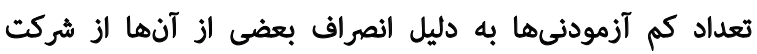

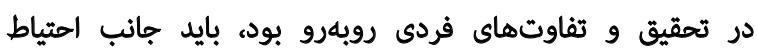
را بيشتر رعايت كرد.

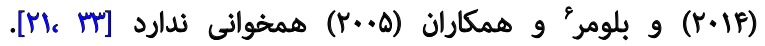

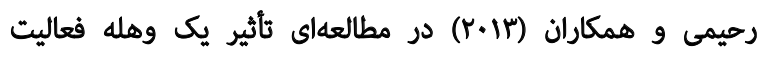

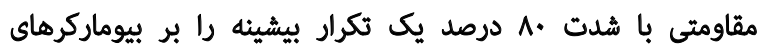

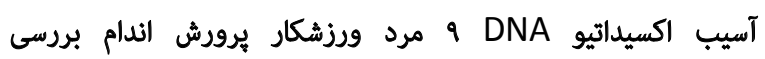
كردند و به اين نتيجه رسيدند كه غلظت OHA

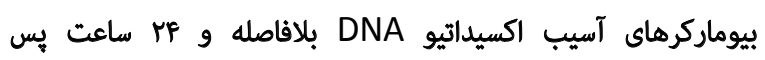

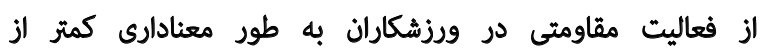

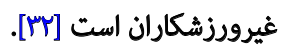

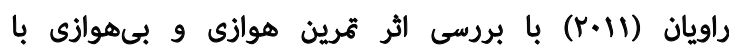

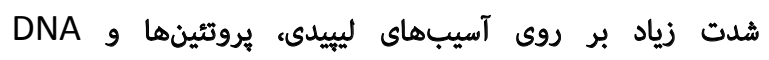

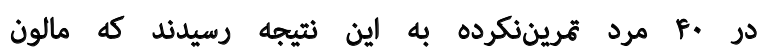

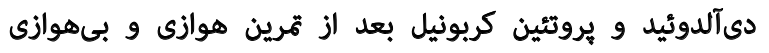

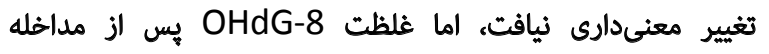

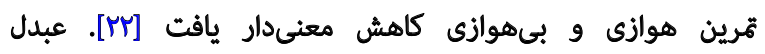

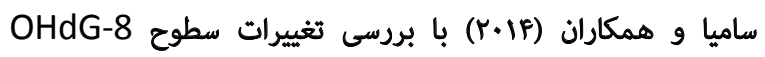

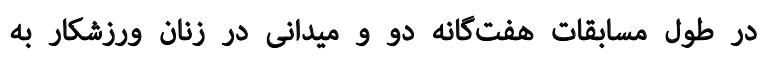

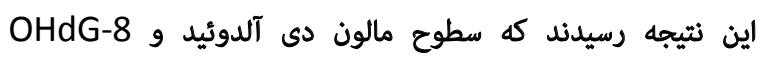

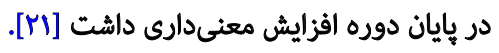

بلومر و همكاران (ه.•r) در مطالعهاى كه اثر تمرينات هوازى

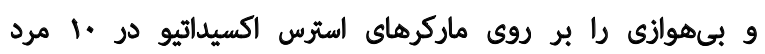

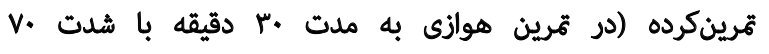

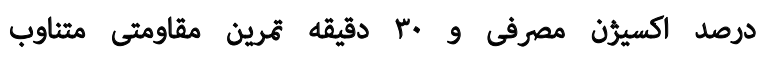

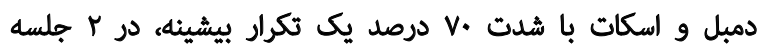

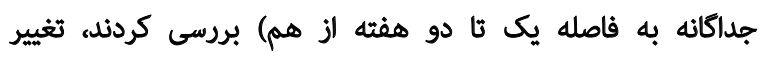

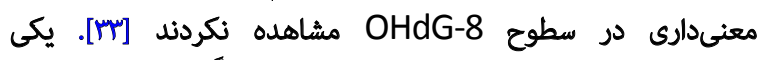

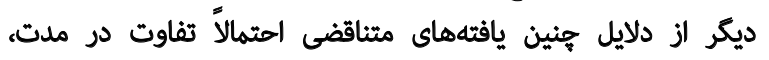

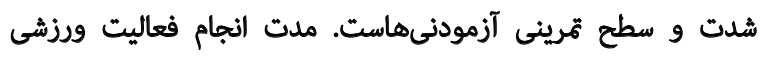

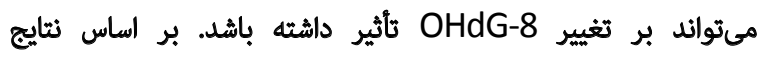

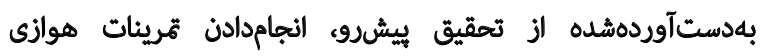

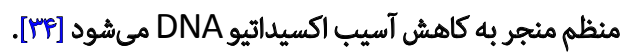

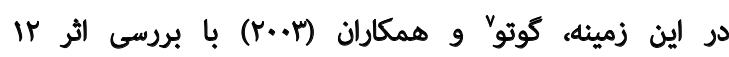

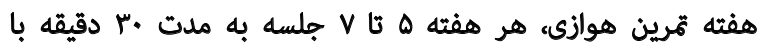

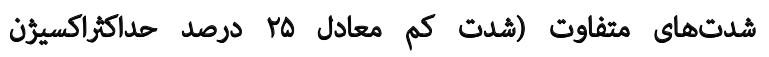

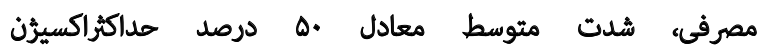

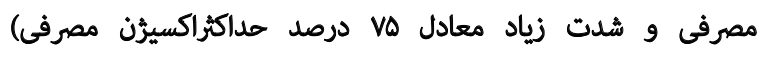

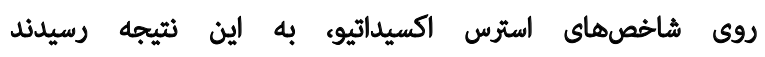
كه غلظت OHdG-8 در شدت زياد افزايش معثى ائدار يافت، اما در شدت متوسط هر دو متغير OHdG-8

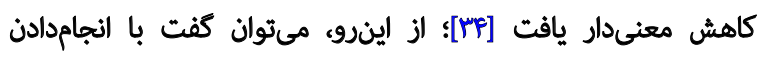

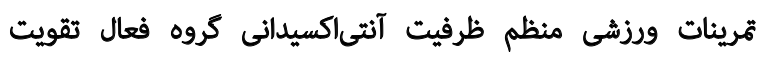

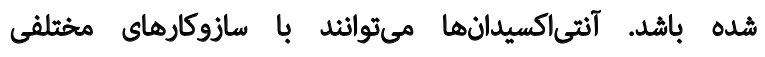




\section{مالاحظات اخلاقى}

ييروى أو اصول اخلاق ثئوهش

اين مقاله از طرح تحقيقاتى ثبتشده در دانشكاه فردوسى

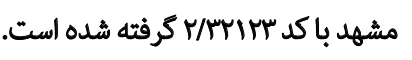

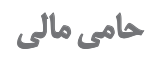

اين طرح با حمايت مالى معاونت برُوهش و فناورى دانشكاه

فردوسى مشهد انجام شد.

$$
\text { مشاركت ثويسند منان }
$$

مفهومسازى: كيوان مجازي، مهدى قهرمانى مقدم، تيمور

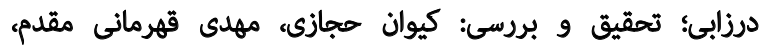

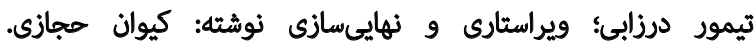

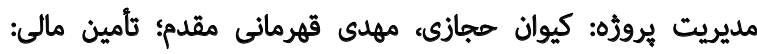

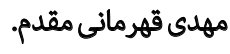

$$
\text { تعارض منافع }
$$

بنا بر اظهارنظر نويسندكان، تعارض منافعى وجود ندارد. 


\section{References}

[1] Hosseini SA, Abdollahi AA, Behnampour N, Salehi A. [The relationship between coronary risk factors and coronary artery involvement based on angiogrpahy findings (Persian)]. Koomesh. 2012; 14(1):7-12.

[2] Buchan D, Ollis S, Thomas N, Baker J. The influence of a high intensity physical activity intervention on a selection of health related outcomes: An ecological approach. BMC Public Health. 2010; 10:8. [DOI:10.1186/1471-2458-10-8] [PMID] [PMCID]

[3] Sharifirad G, Mohebbi S, Matlabi M. [The relationship of physical activity in middle age and cardiovascular problems in old age in retired people in Isfahan, 2006 (Persian)]. Quarterly of Horizon of Medical Sciences. 2007; 13(2):57-63.

[4] Hamedi Nia M, Rezaei S. [The relations of physical activity and body fat percentage to some cardiovascular risk factors in faculty members of Sabzevar teacher training university (Persian)]. Journal of Sabzevar University of Medical Sciences. 2004; 11(3):34-40.

[5] Shamsi A, Ebadi A. [Risk factors of cardiovascular diseases in elderly people (Persian)]. Iranian Journal of Critical Care Nursing. 2011;3(4):183-8.

[6] Christopher P, Cannon M. Cardiovascular disease and modifiable cardiometabolic risk factors. Clinical Cornerstone. 2007; 8(3):11-28. [DOI:10.1016/S1098-3597(07)80025-1]

[7] Mosca L, Banka CL, Benjamin EJ, Berra K, Bushnell C, Dolor RJ, et al. Evidence based guide lines for cardiovascular disease prevention in woman. Circulation. 2007; 11(5):1481-501. [DOI:10.1161/ CIRCULATIONAHA.107.181546] [PMID]

[8] Young I, Woodside J. Antioxidants in health and disease. Journal of Clinical Pathology. 2001; 54(3):176-86. [DOI:10.1136/ jcp.54.3.176] [PMID] [PMCID]

[9] Furukawa S, Fujita T, Shimabukuro M, Iwaki M, Yamada Y, Nakajima $Y$, et al. Increased oxidative stress in obesity and its impact on metabolic syndrome. Journal of Clinical Investigation. 2004; 114(12):1752-61. [DOI:10.1172/JCI21625] [PMID] [PMCID]

[10] Wu LL, Chiou CC, Chang PY, Wu JT. Urinary OHdG-8: A marker of oxidative stress to DNA and a risk factor for cancer, atherosclerosis and diabetics. Clinica Chimica Acta. 2004; 339(1-2):1-9. [DOI:10.1016/j.cccn.2003.09.010] [PMID]

[11] Powers SK, Jackson MJ. Exercise-induced oxidative stress: Cellular mechanisms and impact on muscle force production. Physiological Reviews. 2008; 88(4):1243-76. [DOI:10.1152/physrev.00031.2007] [PMID] [PMCID]

[12] Cunningham P, Geary M, Harper R, Pendleton A, Stover S. High intensity sprint training reduces lipid peroxidation in fasttwitch skeletal muscle. JEP online Submission Guidelines. 2005; 8(6):18-25.

[13] Elosua R, Molina L, Fito M, Arquer A, Sanchez-Quesada J, Covas $\mathrm{M}$, et al. Response of oxidative stress biomarkers to a 16week aerobic physical activity program, and to acute physical activity, in healthy young men and women. Atherosclerosis. 2003; 167(2):327-34. [DOI:10.1016/S0021-9150(03)00018-2]

[14] Dekany M, Nemeskeri V, Györe I, Harbula I, Malomsoki J, Pucsok J. Antioxidant status of interval-trained athletes in various sports. International Journal of Sports Medicine. 2006; 27(2):112-6. [DOI:10.1055/s-2005-865634] [PMID]
[15] Van Guilder GP, Westby CM, Greiner JJ, Stauffer BL, DeSouza CA. Endothelin-1 vasoconstrictor tone increases with age in healthy men but can be reduced by regular aerobic exercise. Hypert. 2007; 50(2):403-9. [DOI:10.1161/HYPERTENSIONAHA.107.088294] [PMID]

[16] Soltis EE. Effect of age on blood pressure and membrane-dependent vascular responses in the rat. Circulation Research. 1987; 61(6):889-97. [DOI:10.1161/01.RES.61.6.889] [PMID]

[17] Rodriguez-Pascual F, Busnadiego O, Lagares D, Lamas S. Role of endothelin in the cardiovascular system. Pharmacological Research. 2011; 63(6):463-72. [DOI:10.1016/j. phrs.2011.01.014] [PMID]

[18] De Bree A, Verschuren WM, Blom HJ, Kromhout D. Lifestyle factors and plasma homocysteine concentrations in a general population sample. American Journal of Epidemiology. 2001; 154(2):150-4. [DOI:10.1093/aje/154.2.150] [PMID]

[19] Kelley GA, Kelley KS. Efficacy of aerobic exercise on coronary heart disease risk factors. European Journal of Preventive Cardiology. 2008; 11(1):71-5. [DOI:10.1111/j.17517141.2008.08037.x] [PMID]

[20] Jarrete A. Influence of aerobic exercise training on cardiovascular and endocrine-inflammatory biomarkers in hypertensive postmenopausal womenq. Journal of Clinical \& Translational Endocrinology. 2014; 1(3):108-14. [DOI:10.1016/j.jcte.2014.07.004] [PMID] [PMCID]

[21] Samia BAA, Youssef GA. Changes in urinary 8-hydroxydeoxyguanosine levels during heptathlon race in professional female athletes. Journal of Human Kinetics. 2014; 41(1):107-11. [DOI:10.2478/hukin-2014-0038] [PMID] [PMCID]

[22] Revan S. Effects of acute high-intensity aerobic and anaerobic exercise on oxidative damage to lipids, proteins and DNA in untrained subjects. African Journal of Pharmacy and Pharmacology. 2011; 5(10):1321-6. [DOI:10.5897/AJPP11.456]

[23] Nojima H, Watanabe H, Yamane K, Kitahara Y, Sekikawa K, Yamamoto $\mathrm{H}$, et al. Effect of aerobic exercise training on oxidative stress in patients with type 2 diabetes mellitus. Metabolism. 2008; 57(2):170-6. [DOI:10.1016/j.metabol.2007.08.021] [PMID]

[24] Friedenreich CM, Pialoux V, Wang Q, Shaw E, Brenner DR, Waltz $X$, et al. Effects of exercise on markers of oxidative stress: An ancillary analysis of the alberta physical activity and breast cancer prevention trial. BMJ Open Sport \& Exercise Medicine. 2016; 2(1):e000171. [DOI: 10.1136/bmjsem-2016-000171]

[25] Thomas S, Reading J, Shephard RJ. Revision of the Physica Activity Readiness Questionnaire (PAR-Q). Canadian Journal of Sport Sciences. 1992; 17(4):338-45. [PMID]

[26] Abdolmaleki Z, Saleh Sedghpour B, Bahram A, Abdolmaleki F. Validity and reliability of the physical self-description questionnaire among adolescent girls. Journal of Applied Psychology. 2011; 4(16):42-55.

[27] Pour-Abdi K, Shakerian S, Pour-Abdi Z, Janbozorgi M. Effects of short-term interval training courses on fitness and weight loss of untrained girls. Annals of Applied Sport Science. 2013; 1(2):1-9.

[28] Fakourian A, Azarbaijani M, Peeri M. Effect a period of selective military training on physical fitness, body mass index, mental health and mood in officer students. Journal of Military Medicine. 2012; 10(1):17-27. 
[29] Dashti M. The effect of programmed exercise on body compositions and heart rate of 11-13 years-old male students. Zahedan Journal of Research in Medical Sciences. 2011; 13(6):40-3.

[30] Horowitz JF, Klein S. Lipid metabolism during endurance exercise. The American Journal of Clinical Nutrition. 2000; 72(2):55863s. [DOI:10.1093/ajcn/72.2.558S] [PMID]

[31] Thomas GA, Cartmel B, Harrigan M, Fiellin M, Capozza S, Zhou $Y$, et al. The effect of exercise on body composition and bone mineral density in breast cancer survivors taking aromatase inhibitors. Obesity. 2017; 25(2):346-51. [DOI:10.1002/oby.21729] [PMID] [PMCID]

[32] Rahimi R, Sharafi H. [The effect of a bout of resistance exercise on 8-Hydroxy-2'-Deoxyguanosine in athletes and non-athletes (Persian)]. Knowledge \& Health. 2012; 7(1):1-7.

[33] Bloomer RJ, Goldfarb AH, Wideman L, McKenzie MJ, Consitt LA. Effects of acute aerobic and anaerobic exercise on blood markers of oxidative stress. The Journal of Strength \& Conditioning Research. 2005; 19(2):276-85. [DOI:10.1519/00124278200505000-00007]

[34] Goto C, Higashi Y, Kimura M, Noma K, Hara K, Nakagawa $\mathrm{K}$, et al. Effect of different intensities of exercise on endotheliumdependent vasodilation in humans. Circulation. 2003; 108(5):5305. [DOI:10.1161/01.cir.0000080893.]

[35] Ranjbar A. [Propofol: A decreaser or increaser drug of oxidative stress: A review (Persian)]. Pajouhan Scientific Journal. 2012; 11(1):1-5.

[36] Clanton TL. Hypoxia-induced reactive oxygen species formation in skeletal muscle. Journal of Applied Physiology. 2007; 102(6):2379-88. [DOI: 10.1152/japplphysiol.01298.2006]

[37] Steinberg JG, Delliaux S, Jammes Y. Reliability of different blood indices to explore the oxidative stress in response to maximal cycling and static exercises. Clinical Physiology and Functional Imaging. 2006; 26(2):106-12. [DOI: 10.1111/j.1475-097x.2006.00658]

[38] Dekleva M, Lazic JS, Pavlovic-Kleut M, Mazic S, Stevanovic A, Soldatovic I, et al. Cardiopulmonary exercise testing and its relation to oxidative stress in patients with hypertension. Hypertension Research. 2012; 35(12):1145-51. [DOI: 10.1038/hr.2012.115]

[39] Shin YA, Lee JH, Song W, Jun TW. Exercise training improves the antioxidant enzyme activity with no changes of telomere length. Mechanisms of Ageing and Development. 2008; 129(5):254-60. [DOI: 10.1016/j.mad.2008.01.001]

[40] Pourfazeli B, Azamian Jazi A, Faramarzi M, Mortazavi MJ. [Effect of regular aerobic exercise on oxidative damage markers of lipids and proteins in rats exposed to radiation emitted by WiFi router (Persian)]. Journal of Torbat Heydariyeh University of Medical Sciences. 2017; 5(2):11-9. 
This Page Intentionally Left Blank 